\title{
TICs Aplicadas a la Enseñanza del Aparato Digestivo
}

\author{
TICs Applied to Teaching the Digestive Apparatus
}

"Rubén Daniel Algieri; ${ }^{* *}$ Martín Javier Mazzoglio y Nabar; ${ }^{* * *}$ Claudia Gabriela Dogliotti \& ${ }^{* * * * *}$ Andrea Gazzotti

\begin{abstract}
ALGIERI, R. D.; MAZZOGLIO Y NABAR, M. J.; DOGLIOTTI, C. G. \& GAZZOTTI, A. TICs aplicadas a la enseñanza del aparato digestivo. Int. J. Morphol., 27(4):1261-1268, 2009.

RESUMEN: Las Tecnologías de la Información y de la Comunicación (TICs) son tecnologías digitales para almacenamiento, transmisión y recepción de informaciones. La sociedad cambió y la educación universitaria debe adecuarse a la presencia de la informática y otras TICs. Las formas de estudio y abordajes del sistema digestivo, sumados a la importancia de sus relaciones anatómicas, lo constituyen un vasto campo para la implementación de TICs. El objetivo de este trabajo fue evaluar la importancia de las TICs como herramienta pedagógica en la enseñanza del sistema digestivo a alumnos universitarios, y correlacionarla con características socioeconómicas y educativas. Se realizó una encuesta estandarizada a 305 alumnos universitarios (ciclo 2007) de la asignatura Anatomía (UBA) y se estudiaron los datos con pruebas estadísticas. El 89,1\% considera útil las TICs durante los trabajos prácticos del sistema digestivo (TPSD), prefiriendo a las presentaciones Power Point ${ }^{\circledR}(68,1 \%)$. El 78\% consideró los casos clínico-quirúrgicos necesarios para fijar conocimientos y mantener la atención en los TPSD. El 82\% utiliza el Museo Virtual como una herramienta de aprendizaje y estudio. Los alumnos que no trabajan manifestaron mayor adherencia a los casos clínico-quirúgicos mientras que los otros eligieron herramientas que maximizasen su tiempo de estudio y fijasen conocimientos. Observamos una correlación $(\mathrm{R} 2=0,72)$ entre las horas trabajadas y la utilización de TICs. Las TICs son la base para un entorno nuevo en el que tendrán que desenvolverse los procesos de enseñanza y aprendizaje. Se evidencia la necesidad de fomentar una concepción "integradora-educacional" de las TICs.
\end{abstract}

PALABRAS CLAVE: Tecnologías de la Información y de la Comunicación; Aparato digestivo; Enseñanza; Aprendizaje.

\section{INTRODUCCIÓN}

Entendemos por Tecnologías de la Información y de la Comunicación (TICs) a las tecnologías digitales utilizadas para el almacenamiento, tratamiento, transmisión y recepción de informaciones y mensajes (Gros, 2000; Levis, 2006; Levis, 2007; Skinner, 1958). La sociedad en la que se desenvuelve la educación universitaria no es la misma en la que fue creada, por lo cual su metamorfosis es necesaria y hasta obligada. La educación en sus diferentes modalidades y niveles debe adaptarse a la presencia de la informática y de otras TICs en la mayoría de sus actividades (Brunner, 2003; Levis, 2006; Marenco \& Urvoy, 1975).

El ingreso masivo de computadoras y dispositivos informáticos en actividades educativas condiciona la con- ceptualización, el pensamiento y hasta la memorización de experiencias y conocimientos, influyendo en la resolución futura de tareas (De Kerckhove, 1999; Skinner). Diversos autores señalan que la presencia de TICs no implica que hayan cambiado de manera significativa las prácticas áulicas y que su uso responda a propuestas pedagógicas innovadoras y transformadoras. Las TICs han reabierto debates sobre las formas de enseñanza y sobre cómo otros espacios (incluyendo los virtuales) son un soporte para el aprendizaje (Brunner).

En relación con el sistema digestivo, las múltiples formas de estudio (diagnósticos y reconstrucciones 3D) y abordajes, sumados a la importancia en el conocimiento de

Médico, UBA. Especialista en Cirugía General. Jefe de Servicio de Cirugía, Hospital Aeronáutico Central. Profesor Adjunto de Anatomía e Histología, UM. Jefe Trabajos Prácticos de Anatomía, III Cátedra de Anatomía, Universidad de Buenos Aires, Argentina.

* Médico, UBA. Residente de Psiquiatría. Docente Auxiliar de Anatomía, III Cátedra de Anatomía, UBA. Docente Auxiliar de Farmacología, III Cátedra de Farmacología. Monitor de Investigaciones Clínicas y Farmacológicas, Universidad de Buenos Aires, Argentina.

*** Médica, UBA. Especialista en Psiquiatría. Jefe de Trabajos Prácticos de Anatomía, III Cátedra de Anatomía, Universidad de Buenos Aires, Argentina.

***** Médica, UBA. Especialista en Medicina Física y Rehabilitación. Jefe de Trabajos Prácticos de Anatomía, III Cátedra de Anatomía, Universidad de Buenos Aires, Argentina. 
las relaciones anatómicas, lo constituyen como un vasto campo para la implementación de TICs en su enseñanza.

Actualmente asistimos a la transición entre dos paradigmas pedagógicos referentes al modo de enseñanza y aprendizaje con TICs: el instrumental-utilitario que propugna la utilización de las mismas como recurso didáctico a modo de "máquinas de enseñar" y complementariamente como "bibliotecas de información"; y el "integrador-educacional" que asigna un rol activo al enseñar y aprender basado en la exploración, experimentación, debate y reflexión, propone que las TICs sean desarrolladas como prácticas pedagógicas innovadoras (proyectos en red, formas bimodales de clase; uso de materiales hipermedia y simulaciones) (Levis, 2007; Marenco \& Urvoy).

El objetivo fue evaluar la importancia de las TICs como herramienta pedagógica en la enseñanza del sistema digestivo a alumnos universitarios. Asimismo, correlacionar su importancia educativa en relación con las características socioeconómicas y educativas de los encuestados.

\section{MATERIAL Y MÉTODO}

Se realizó en diciembre de 2007 una encuesta estandarizada y normatizada a 305 alumnos universitarios de ciclo 2007 de la asignatura Anatomía Humana de la Facultad de Medicina de la Universidad de Buenos Aires, Argentina. Las características poblacionales de la muestra con sus resultados se exhiben en la Tabla I.

Se realizó el análisis estadístico sobre los datos obtenidos extrayendo el promedio, máximo, mínimo, moda, mediana, desvío estándar (DS) y los coeficientes de correlación (r y R2).

\section{RESULTADOS}

El resultado cuantitativo y porcentual a las preguntas realizadas se exhibe en la tabla II. El rango de edad de los encuestados es de 19 a 42 años (promedio=20,5 años; DS $=2,04)$, siendo de sexo femenino $(68,8 \%)$ y argentinos $(93,1 \%)$. Refirió el $69,1 \%$ haber asistido a escuela privada y realizado el CBC (Ciclo Básico Común) en 1 año el 52.3\%, considerándolo útil para su formación universitaria el 56,6\%. Sólo el 25\% de loa encuestados trabaja, de los cuales el $40,8 \%$ lo hace 5 días a la semana, y el $71,1 \%$ entre 4 y 8 horas diarias. El 77,6\% de los encuestados refirió que era su primera vez cursando la materia y el $35 \%$ tenía las otras materias en curso. El 32,6\% refiere dedicarle menos de 15 horas al estudio de Anatomía, y sólo el 18,8\% le dedica más de 25 horas.

El 89,1\% considera útil la utilización de recursos pedagógicos para complementar el material cadavérico durante los trabajos prácticos del sistema digestivo (TPSD), señalando a las presentaciones en Power Point ${ }^{\circledR}$ y al acceso a Internet como los más provechosos $(68,1 \%$ y $52 \%$ respectivamente). Asimismo, el $83.9 \%$ consideró que la implementación de casos clínico-quirúrgicos referidos al TPSD son necesarios puesto sirven para fijar conocimientos $(78,3 \%)$ y mantener la atención durante el TPSD $(78 \%)$. Los encuestados señalaron que para lograr mayores niveles de atención en el TP elegirían las presentaciones de imágenes en Power Point ${ }^{\circledR}$ (69,7\%) y los casos clínicos como los más eficaces (53\%). Más del 82\% de los encuestados adhiere a la utilización del Museo Virtual de Anatomía considerándolo útil por ser una herramienta de ayuda en su proceso de aprendizaje y estudio y una extensión del proceso educativo más allá de los límites físicos de la Universidad. El 93,8\% de los encuestados eligió los teóricos con presentaciones especiales y virtuales como método de cursada, en contraste con los teóricos o teórico-prácticos.

Los encuestados provenientes de escuelas públicas manifestaron una mayor elección por recursos pedagógicos clásicos, tangibles y pragmáticos con vistas a su futura profesión médica, en contraste a los de escuelas privadas que prefirieron como herramientas las TIC y los espacios virtuales poniendo énfasis en el razonamiento de los conocimientos impartidos (Figs. 1, 2 y 3).

La población de alumnos que no trabaja refirió una mayor adherencia a los casos clínico-quirúgicos por fijar mejor los conocimientos y no consideraron ni muy útil ni una herramienta pedagógica a los espacios virtuales (Museo Virtual). En contraste, los encuestados que trabajan eligieron herramientas que maximizasen su tiempo de estudio, que fuesen eficaces para fijar conocimientos (por vía visual, oral y práctica) y mantener la atención en los TPSD (Figs. 4 y 5).

Se observó una correlación positiva $(\mathrm{R} 2=0,72)$ entre el aumento de la cantidad de horas trabajadas y la utilización de las TIC por parte de los encuestados (Figura 7). Y se determinó que el mayor uso de TIC era inversamente proporcional proporcional a las horas de estudio empleadas ( $\mathrm{r}=-$ $0,78)$.

En el presente trabajo no se cuantificó el nivel de aprendizaje de los sujetos encuestados producto del uso de TICs. 
ALGIERI, R. D.; MAZZOGLIO Y NABAR, M. J.; DOGLIOTTI, C. G. \& GAZZOTTI, A. TICs aplicadas a la enseñanza del aparato digestivo. Int. J. Morphol., 27(4):1261-1268, 2009.

Tabla I. Características poblacionales de la muestra.

\begin{tabular}{|c|c|c|c|}
\hline \multirow[t]{4}{*}{ Edad } & 19 años & 80 & $26,3 \%$ \\
\hline & 20 años & 105 & $34,5 \%$ \\
\hline & 21 años & 68 & $22,4 \%$ \\
\hline & $>21$ años & 51 & $16,7 \%$ \\
\hline \multirow[t]{3}{*}{ Sexo } & Masculino & 93 & $30,6 \%$ \\
\hline & Femenino & 209 & $68,8 \%$ \\
\hline & $\mathrm{N} / \mathrm{C}$ & 2 & $0,7 \%$ \\
\hline \multirow[t]{3}{*}{ Origen } & Argentino & 283 & $93,1 \%$ \\
\hline & No Argentino & 7 & $2,3 \%$ \\
\hline & $\mathrm{N} / \mathrm{C}$ & 14 & $4,6 \%$ \\
\hline \multirow[t]{3}{*}{ Concurrió a colegio } & Privado & 210 & $69,1 \%$ \\
\hline & Público & 84 & $27,6 \%$ \\
\hline & $\mathrm{N} / \mathrm{C}$ & 10 & $3,3 \%$ \\
\hline \multirow[t]{4}{*}{ Cantidad años en que realizó el CBC } & 1 & 159 & $52,3 \%$ \\
\hline & 1,5 & 42 & $13,8 \%$ \\
\hline & 2 & 75 & $24,7 \%$ \\
\hline & $>2$ & 22 & $7,2 \%$ \\
\hline \multirow[t]{3}{*}{ Considera útil el CBC } & Sí & 172 & $56,6 \%$ \\
\hline & No & 119 & $39,1 \%$ \\
\hline & $\mathrm{N} / \mathrm{C}$ & 13 & $4,3 \%$ \\
\hline \multirow[t]{3}{*}{ ¿Trabaja? } & Sí & 76 & $25,0 \%$ \\
\hline & No & 225 & $74,0 \%$ \\
\hline & $\mathrm{N} / \mathrm{C}$ & 3 & $1,0 \%$ \\
\hline \multirow[t]{3}{*}{ ¿Cuantas horas al día? } & $<4$ & 4 & $5,3 \%$ \\
\hline & $4-8$ & 54 & $71,1 \%$ \\
\hline & $>8$ & 15 & $19,7 \%$ \\
\hline \multirow[t]{3}{*}{ ¿Cuántos días por semana? } & $<5$ & 29 & $38,2 \%$ \\
\hline & 5 & 31 & $40,8 \%$ \\
\hline & $>5$ & 13 & $17,1 \%$ \\
\hline \multirow[t]{3}{*}{ ¿Trabaja simultáneamente mientras estudia? } & No & 202 & $66,4 \%$ \\
\hline & Sí & 48 & $15,8 \%$ \\
\hline & $\mathrm{N} / \mathrm{C}$ & 54 & $17,8 \%$ \\
\hline \multirow[t]{3}{*}{ ¿Primera vez que cursa Anatomía? } & No & 65 & $21,4 \%$ \\
\hline & Sí & 236 & $77,6 \%$ \\
\hline & $\mathrm{N} / \mathrm{C}$ & 3 & $1,0 \%$ \\
\hline \multirow[t]{5}{*}{ ¿Tiene Histología? } & Aprobada & 15 & $4,9 \%$ \\
\hline & Regular & 107 & $35,2 \%$ \\
\hline & Libre & 56 & $18,4 \%$ \\
\hline & En curso & 117 & $38,5 \%$ \\
\hline & $\mathrm{N} / \mathrm{C}$ & 7 & $2,3 \%$ \\
\hline \multirow[t]{5}{*}{ ¿Tiene Salud Mental? } & Aprobada & 54 & $17,8 \%$ \\
\hline & Regular & 113 & $37,2 \%$ \\
\hline & Libre & 13 & $4,3 \%$ \\
\hline & En curso & 91 & $29,9 \%$ \\
\hline & $\mathrm{N} / \mathrm{C}$ & 33 & $10,9 \%$ \\
\hline \multirow[t]{5}{*}{ ¿Cuantas horas semanales le dedica al estudio de la Anatomía? } & $<15$ & 99 & $32,6 \%$ \\
\hline & 15 & 42 & $13,8 \%$ \\
\hline & 20 & 46 & $15,1 \%$ \\
\hline & 25 & 60 & $19,7 \%$ \\
\hline & $>25$ & 57 & $18,8 \%$ \\
\hline
\end{tabular}


Tabla II. Resultado cuantitativo y porcentual de las preguntas realizadas.

¿Considera necesario la utilización en el TP de recursos pedagógicos que complementen el material cadavérico?

Si respondió Sí, ¿Cuál de las siguientes herramientas pedagógicas considera útil?

Presentaciones Power Point

Clase con tiza/marcador/pizarra

Maquetas

Presentaciones de preparaciones especiales

Acceso a Internet durante el TP

Láminas

Considera necesario que se aplique casos clínico-quirúrgicos referente al práctico en desarrollo?

Dichos casos clínicos le sirven

Para fijar mejor los conocimientos

Para entender la importancia del objetivo anatómico en desarrollo

Para obtener un mayor acercamiento con su futura profesión

Para mantener su nivel de atención durante la duración del TP

¿Qué herramientas utilizaría para lograr el mayor nivel de atención durante el TP?

Presentaciones en TV de imágenes en Power Point

Acceso a Internet - Museo Virtual

Láminas

Maquetas 3D

Casos Clínicos

Referente al Museo Virtual de la II Cátedra de Anatomía

¿La considera útil?

¿Cree le ayudaría en su proceso de aprendizaje pedagógico?

¿La considera una herramienta más de estudio?

¿Cree que la existencia de elementos virtuales sirve como extensión del proceso educativo más allá de los físicos?

¿Qué prefiere cursar?

Teóricos

Teóricos con presentaciones especiales o virtuales?

Teóricos - Prácticos

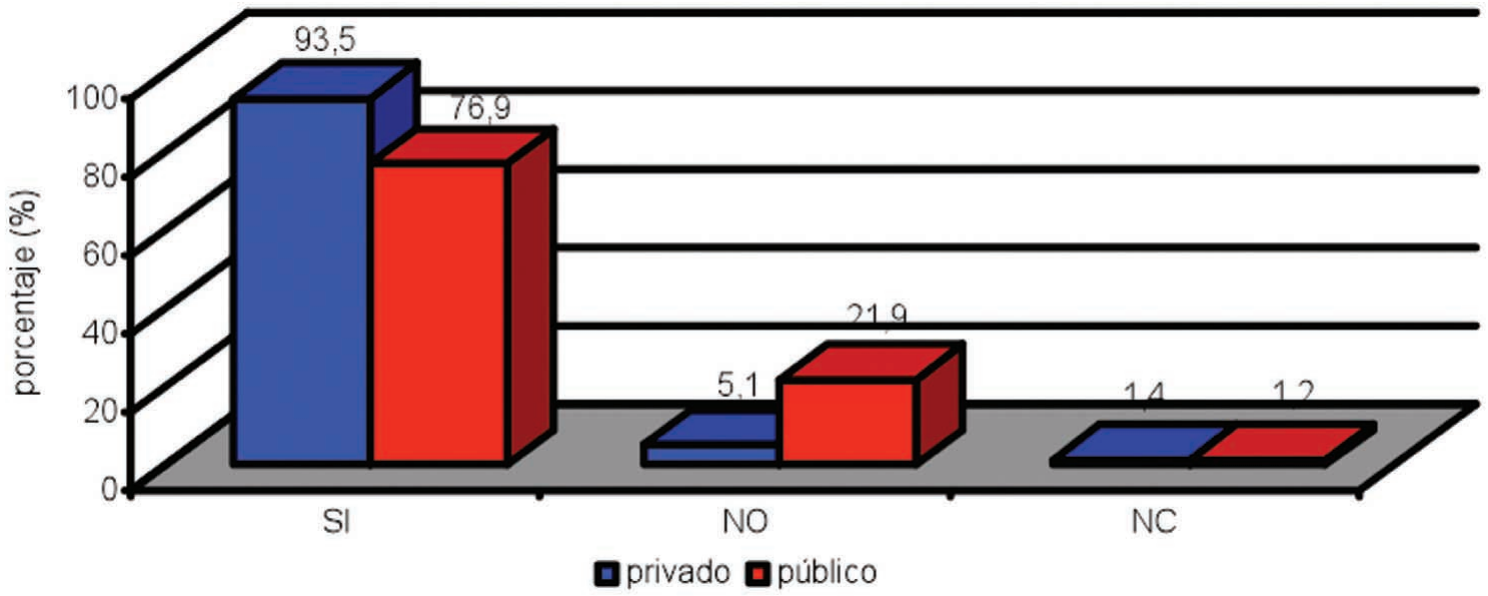

Fig. 1. Porcentaje de respuestas a la necesidad de utilización de recursos pedagógicos en los trabajos prácticos del sistema digestivo. 


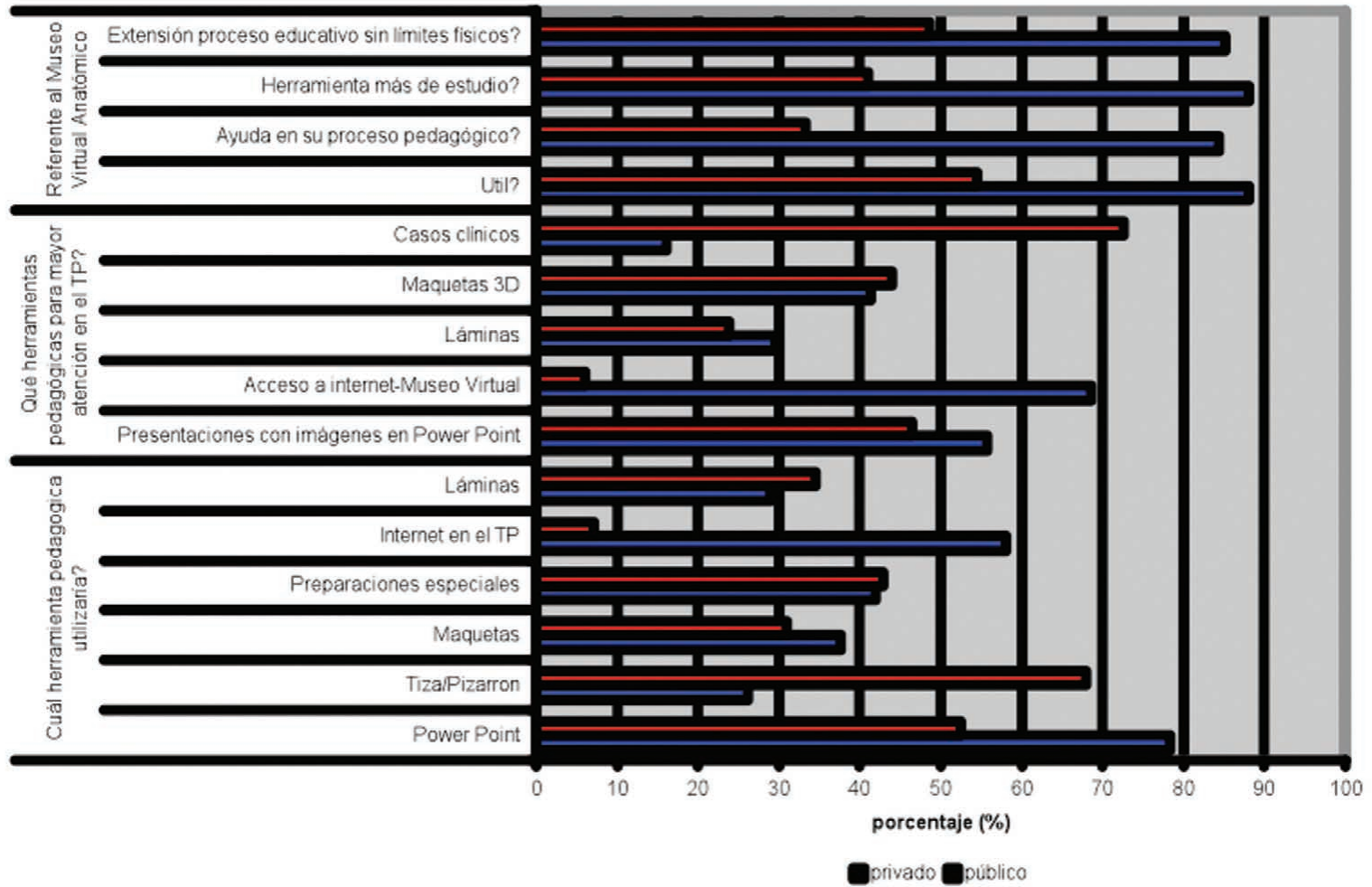

Fig. 2. Porcentaje de respuestas a 3 preguntas formuladas en la encuesta y su discriminación entre alumnos provenientes de escuelas públicas y privadas.

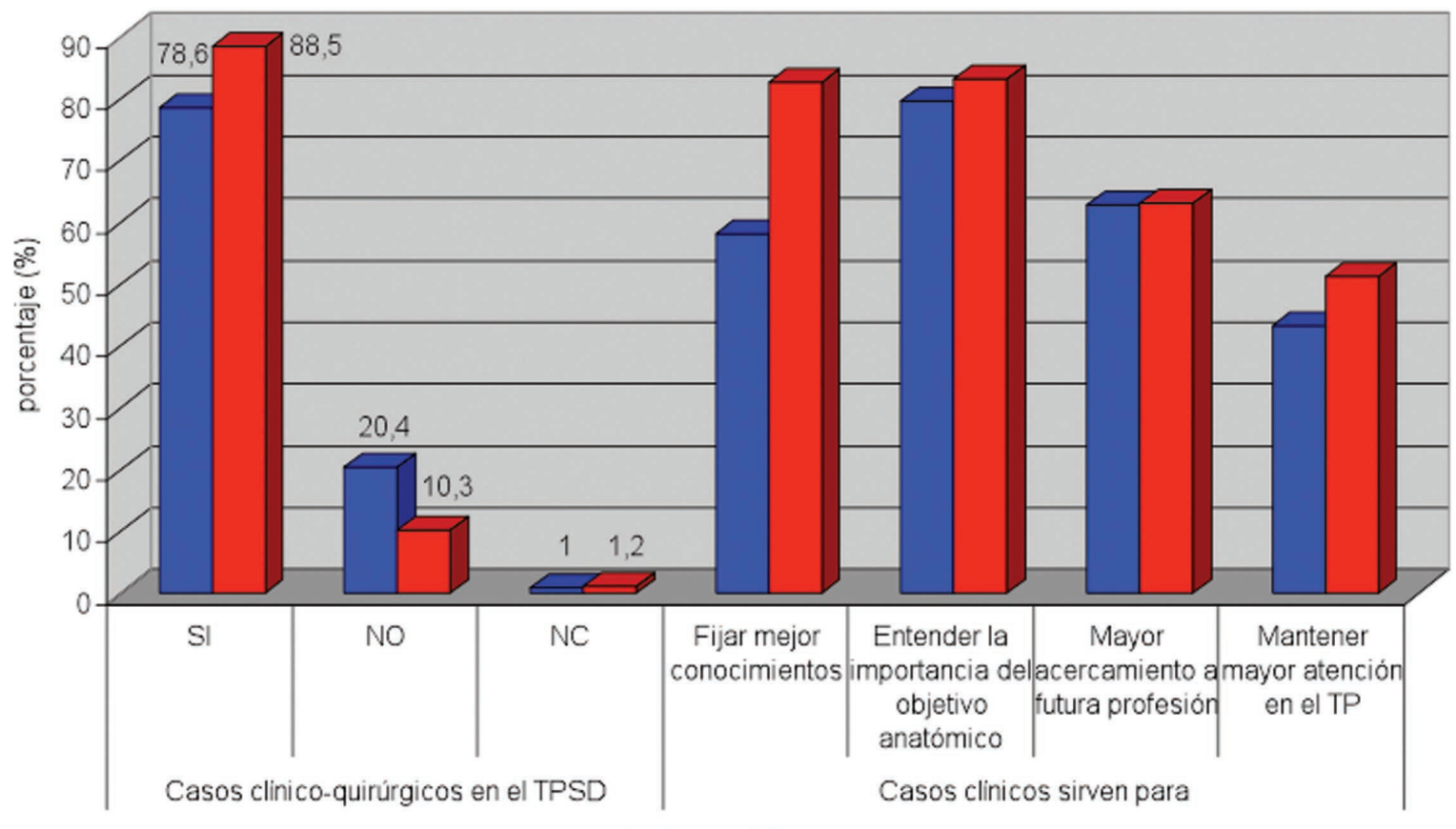

aprivado apúblico

Fig. 3. Porcentaje de respuestas a la implementación de casos clínico-quirúrgicos durante el desarrollo de los trabajos prácticos del sistema digestivo. 


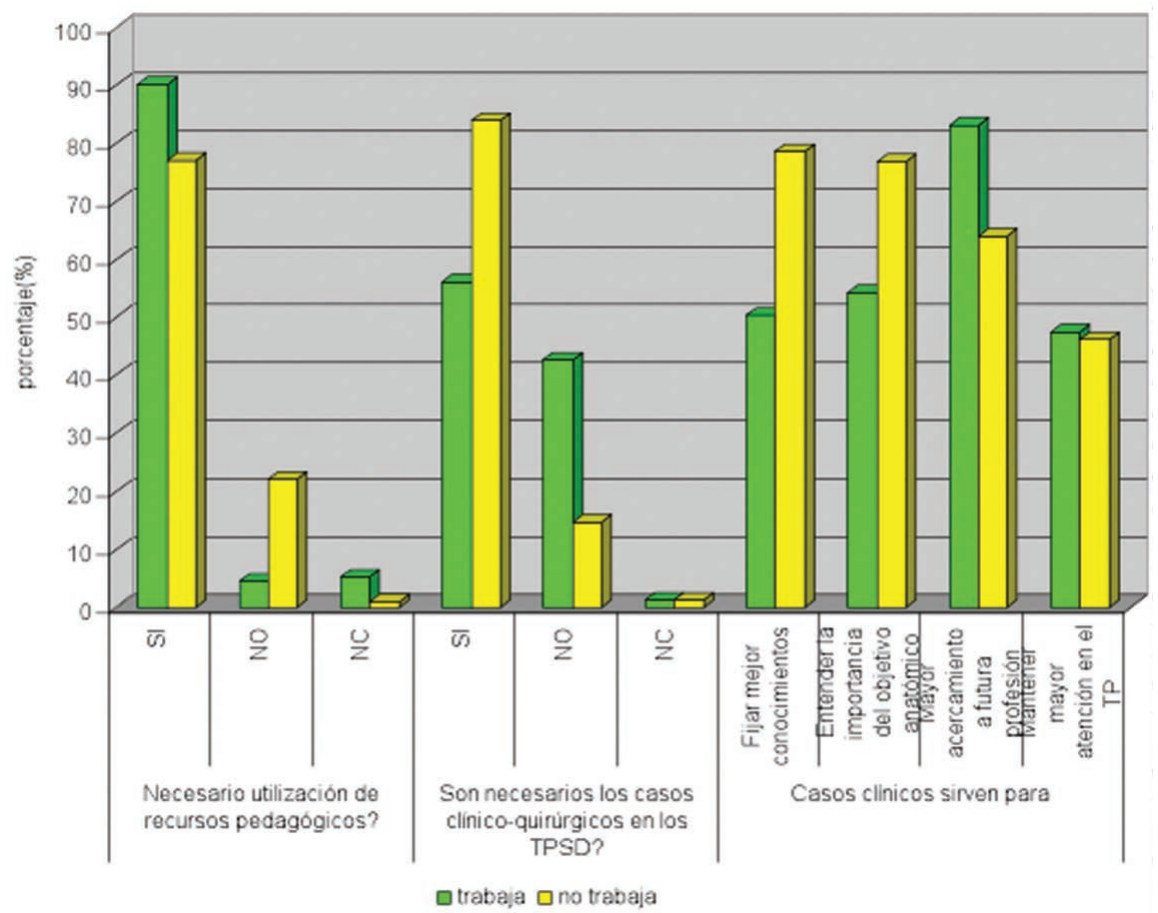

Fig. 4. Porcentaje de respuestas a 3 preguntas formuladas en la encuesta en función de la situación laboral de los encuestados.

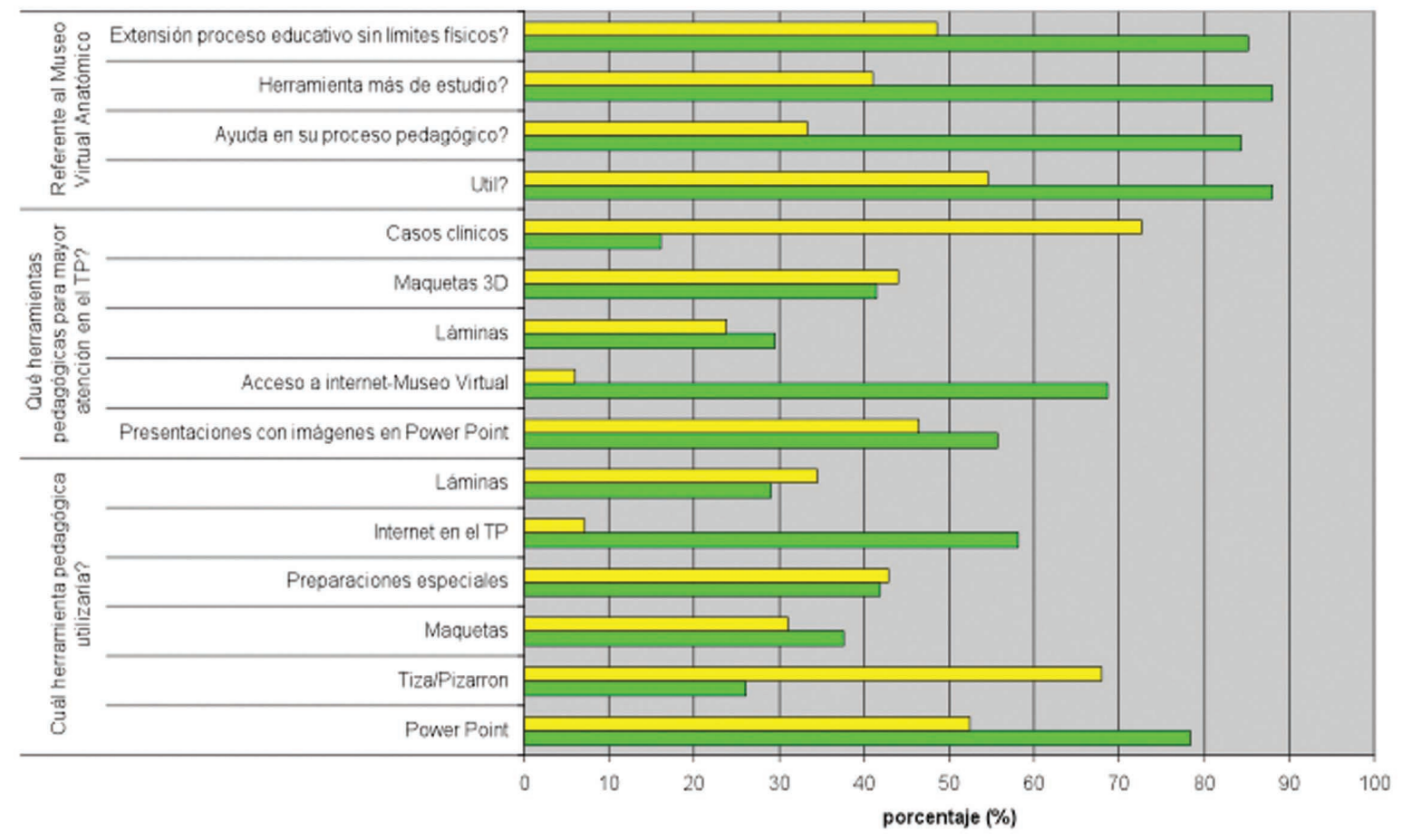

口 trabaja $\square$ no trabaja

Fig. 5. Porcentaje de respuestas a 3 preguntas formuladas en la encuesta en función de la situación laboral de los encuestados. 


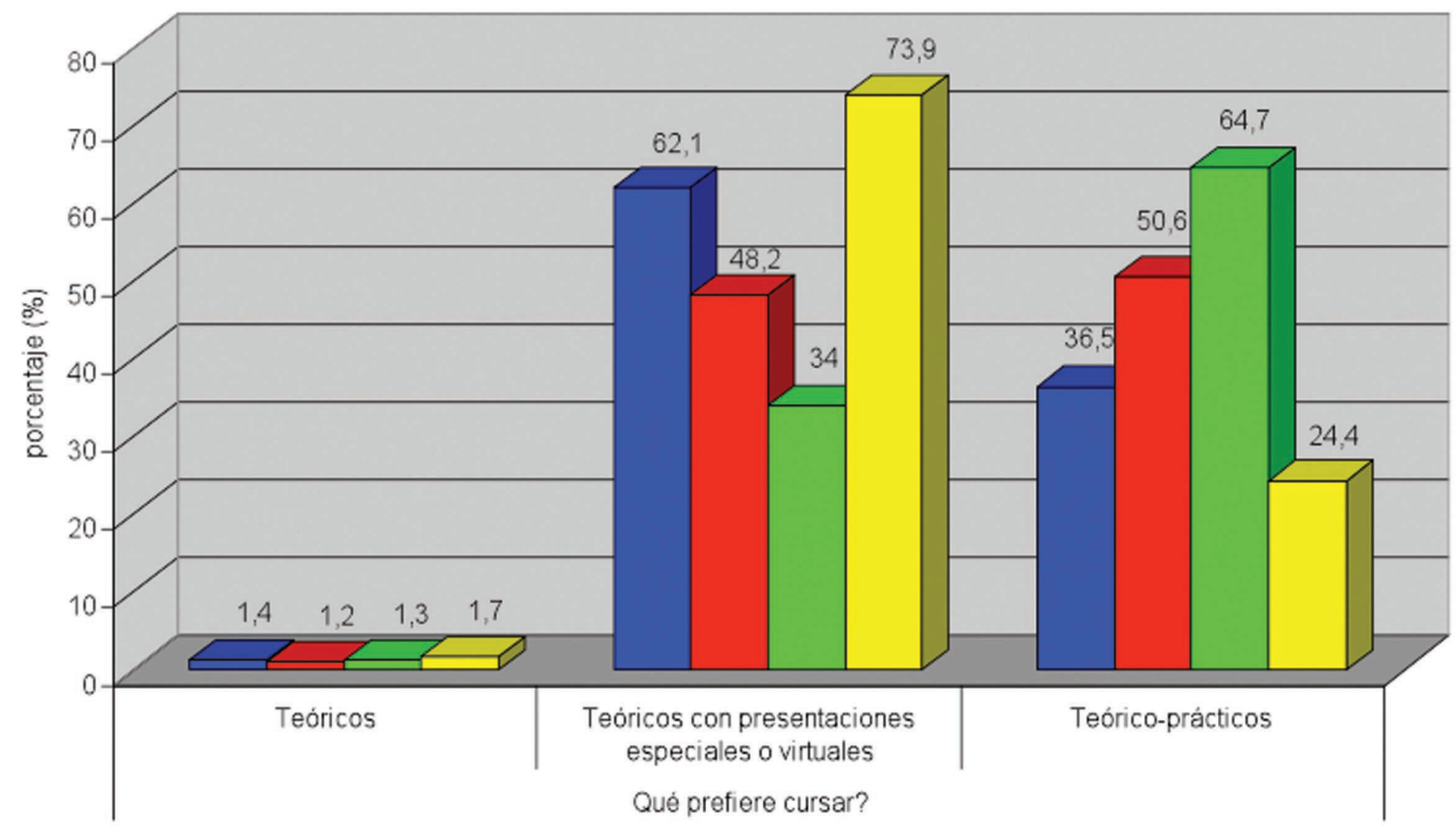

a privado a público utrabaja $\square$ no trabaja

Fig. 6. Porcentaje de respuestas a la preferencia en la modalidad de cursada en función de la situación laboral y del tipo de establecimiento educativo (público o privado).

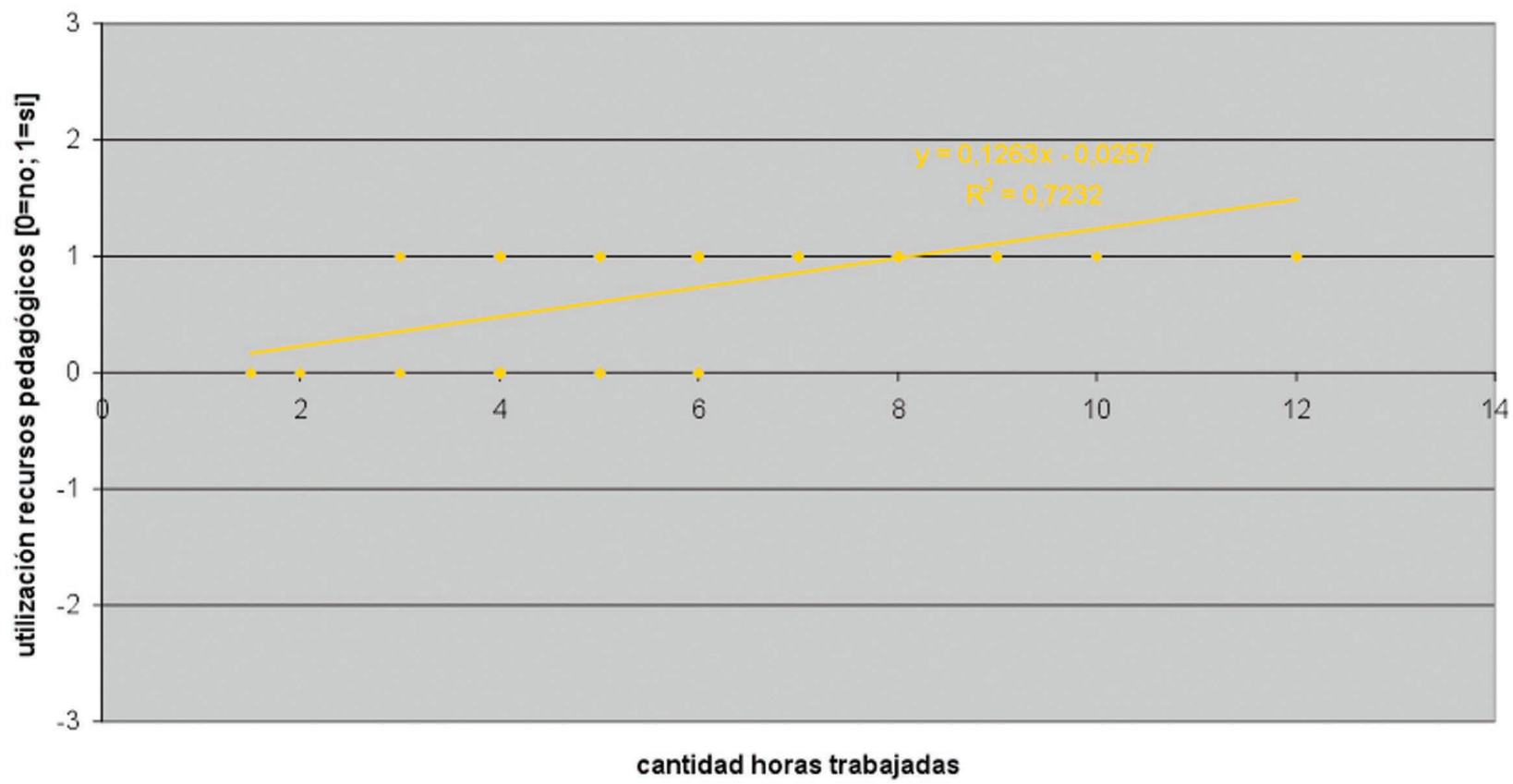

Fig. 7. Correlación entre la cantidad de horas trabajadas y la aceptación en el uso de recursos pedagógicos. 


\section{CONCLUSIONES}

Las TICs son la base para el surgimiento de un entorno nuevo y diferente dentro del cual tendrán que desenvolverse los procesos de enseñanza y aprendizaje. Se evidencia la necesidad de fomentar una concepción "integradora-educacional" de las TICs más que "instrumental-utilitaria", acorde con los requerimientos planteados por los alumnos. En la actualidad, a la transmisión de conocimiento a través del lenguaje se agregaron otros métodos alternativos que obligan al personal docente al conocimiento de nuevas tecnologías para el manejo de la información y la comunicación.

ALGIERI, R. D.; MAZZOGLIO Y NABAR, M. J.; DOGLIOTTI, C. G. \& GAZZOTTI, A. TICs applied to teaching the digestive apparatus. Int. J. Morphol., 27(4):1261-1268, 2009.

SUMMARY: TICs (Technologies of Information and Communication) are digital technologies for saving, transmitting and receiving information. The society has changed and the University education must be updated due to the presence of computers and other TICs. The ways of studying the digestive system, together with the importance of its anatomical relations, makes it a wide field for the use of TICs. The objective of this study were to evaluate the importance of TICs as a pedagogic tools in teaching the digestive system to University students, and connect it with the social, educational and economic characteristics. A standardized poll was done to 305 university students (year 2007) from the course Anatomy (UBA) and the data was studied with statistic tests. 89,1\% finds TICs useful during the digestive system TP., preferring Power Point presentations (68.1\%). 78\% considered the clinical surgical cases necessary to fixate Knowledge and concentrate during the TPSD. $82 \%$ uses the Virtual Museum as a tool for learning and study. The students that don't work showed more adherence to the clinic-surgical cases. Meanwhile, the others chose tools that maximize their study time and fixate knowledge. We saw a correlation $(\mathrm{r} 2=0.72)$ between the amount of hours worked and the use of tics. Tics are a base for a new background in which the process of teaching and learning will have to develop. It can be seen that there is a need to encourage an "educational-integrative" conception of TICs.

KEY WORDS: Technologies of Information and Communication; Digestive apparatus; Teaching; Learning.

\section{REFERENCIAS BIBLIOGRÁFICAS}

Brunner, J. J. Educación e Internet ¿La próxima revolución?. Santiago de Chile, Editorial Fondo de Cultura Económica, 2003.

De Kerckhove, D. Inteligencias en conexión. Hacia una sociedad de la web. $1^{\text {a }}$ Edición. Barcelona, Gedisa Editorial, 1999. pp.45-50.

Gros, B. El ordenador invisible. Hacia la apropiación del ordenador en la enseñanza. $1^{\mathrm{a}}$ Edición. Barcelona, Gedisa Editorial-Biblioteca Educación, 2000.

Levis, D. Alfabetos y saberes: La alfabetización digital. Comun. Rev. Medios Comun. Educ., 26:78-82, 2006.

Levis, D. Enseñar y aprender con informática / Enseñar y aprender informática. Medios informáticos en la escuela argentina. En: Cabello-Levis (editores). Medios informáticos en la educación a principios del siglo XXI. Buenos Aires, Prometeo, 2007. pp.21-50.

Marenco, C. \& Urvoy, J. Informática y sociedad. Barcelona, Editorial Labor SA, 1975.
Skinner, B. F. Teaching Machines. From the experimental study of learning come devices which arrange optimal conditions for self-instruction. Science, 24(158):969-77, 1958.

Dirección para correspondencia:

Martín J. Mazzoglio y Nabar

Aráoz 1778 (C1414DQJ)

Ciudad Autónoma de Buenos Aires

REPÚBLICAARGENTINA.

Email: mazzoglioynabar@hotmail.com

Received: 31-07-2009

Accepted: 03-11-2009 